\title{
Electromagnetic response of closely spaced metal meshes
}

\author{
Melita C. Taylor, Alastair P. Hibbins, and J. Roy Sambles \\ Electromagnetic Materials Group, School of Physics, University of Exeter Stocker Road, Exeter, EX4 4QL, United Kingdom
}

(Received 18 August 2011; revised manuscript received 4 June 2012; published 17 July 2012)

\begin{abstract}
The electromagnetic transmittance of a double layer of identical square arrays of square holes (mesh) in a perfectly conducting sheet is analytically modeled using a modal matching technique. The structure supports families of standing-wave modes together with surface modes that, close to the onset of diffraction, interact with each other. For frequencies below the onset of diffraction, it is the strength of this interaction mediated by evanescent diffraction in the near fields that dictates the electromagnetic response, which is studied as a function of mesh separation and the lateral misalignment between the meshes.
\end{abstract}

DOI: 10.1103/PhysRevB.86.035126

PACS number(s): 42.25.Bs, 41.20.Jb, 42.25.Fx, 78.66.Bz

\section{INTRODUCTION}

There is an extensive and long-standing body of work regarding the response of periodically structured metallic media to electromagnetic (EM) radiation. This spans from Wood's seminal observation in 1902 (Ref. 1) of reflection anomalies from metal gratings in the visible domain, through Ulrich's work in 1967 in the microwave regime, ${ }^{2}$ to 1998 when Ebbesen et al. $^{3}$ observed that a regular square array of subwavelength holes in thin metal screens provided enhanced optical transmission (EOT) at frequencies near the onset of diffraction. This work stimulated a resurgence of interest in this whole field.

It is known that a planar metal dielectric interface can support a surface plasmon polariton (SPP). ${ }^{4}$ However, the momentum of these surface waves is greater than that of the incident photon, and so incident radiation cannot directly couple to them on a planar interface. One method of coupling to the surface mode is via grating coupling, for example, corrugation of the surface as in metal meshes. ${ }^{3,5}$ The periodic meshes studied in the present work also support plasmonlike surface bound modes ${ }^{6,7}$ that can be coupled to by the incident radiation enabling enhanced transmission through the hole array.

A variety of modeling techniques to predict the EM response of these periodically patterned structures have been developed, of which transmission line models, ${ }^{5,8,9}$ finite-element method (FEM) models ${ }^{10}$ and modal-matching methods ${ }^{6,11-16}$ are some examples. In the present study, the modal-matching method employed previously for a single mesh laye ${ }^{17}$ has been extended and applied to a double-layered mesh system. Each mesh layer comprises a two-dimensional (2D) square array of square holes in a sheet of perfect electrical conductor (PEC) of thickness $h$. The two meshes, which share common orthogonal axes along the sides of the square holes, are separated by a dielectric layer of thickness $g$, with $b$ denoting the lateral displacement of the meshes with respect to each other (Fig. 1) along the polarization axis of the normally incident radiation. Investigation of the single mesh layer showed that there is a strong transmission resonance due to a resonantly excited surface wave located at a frequency slightly below the diffraction edge. ${ }^{17}$ Here a double mesh layer is studied with particular emphasis on unraveling the influence of evanescent coupling on the effect on the transmission of translational displacement of one mesh relative to the other.
Although most studies on the EM response of metal meshes have concentrated on single mesh layers, there have been some investigations into double mesh structures. In 2000, Yu et al. created a reflective polarizer using a double layer of metal monogratings at optical frequencies. ${ }^{18}$ Another optical investigation studied double-layer meshes where the small separation between the layers allows coupling of the SPPs supported by each mesh, leading to extraordinary optical transmission even when the layers are laterally misaligned with respect to each other. ${ }^{19}$ The effect of lateral displacement on double mesh layers in the visible regime was studied by Cheng et al. in $2007 .{ }^{20} \mathrm{He}$ et al. conducted an almost identical study to that presented in this paper, albeit at optical frequencies reaching similar conclusions, ${ }^{21}$ as did Miyamaru and Hangyo in the terahertz regime. ${ }^{22} \mathrm{~A}$ common motivation behind the study of double-mesh-layer structures in the optical regime appears to be the creation of negative index materials. ${ }^{18,19,23}$

\section{THEORETICAL FORMALISM}

The theoretical formalism is the same as utilized previously for a single mesh layer, ${ }^{17}$ with the electric fields in the regions above and below the mesh layer described by a two-dimensional Fourier-Floquet expansion of the diffracted orders [Eqs. (1)-(4)] [note that the time-dependent field component,exp $(-i \omega t)$, has been omitted throughout for simplicity, and the relative permeability is taken as unity],

$$
\begin{aligned}
& E_{x}^{\text {diel }}=\sum_{m, n} A_{x}^{m, n} \psi_{1}^{m, n}(x, y) \exp \left(-i k_{z}^{m, n} z\right), \\
& E_{y}^{\text {diel }}=\sum_{m, n} A_{y}^{m, n} \psi_{1}^{m, n}(x, y) \exp \left(-i k_{z}^{m, n} z\right),
\end{aligned}
$$

where

$\psi_{1}^{m, n}(x, y)=\exp \left[i\left(k_{x}+\frac{2 m \pi}{d}\right) x\right] \exp \left[i\left(k_{y}+\frac{2 n \pi}{d}\right) y\right]$

and

$k_{z}^{m, n}=\sqrt{\varepsilon_{\mathrm{d}}\left(\frac{\omega}{c}\right)^{2}-\left(k_{x}+\frac{2 m \pi}{d}\right)^{2}-\left(k_{y}+\frac{2 n \pi}{d}\right)^{2}}$.

The diffracted orders are designated by the integers $m$ and $n$, the wave vector of the diffracted order by $k$, the period of the array by $d$, the relative permittivity of the region between the 


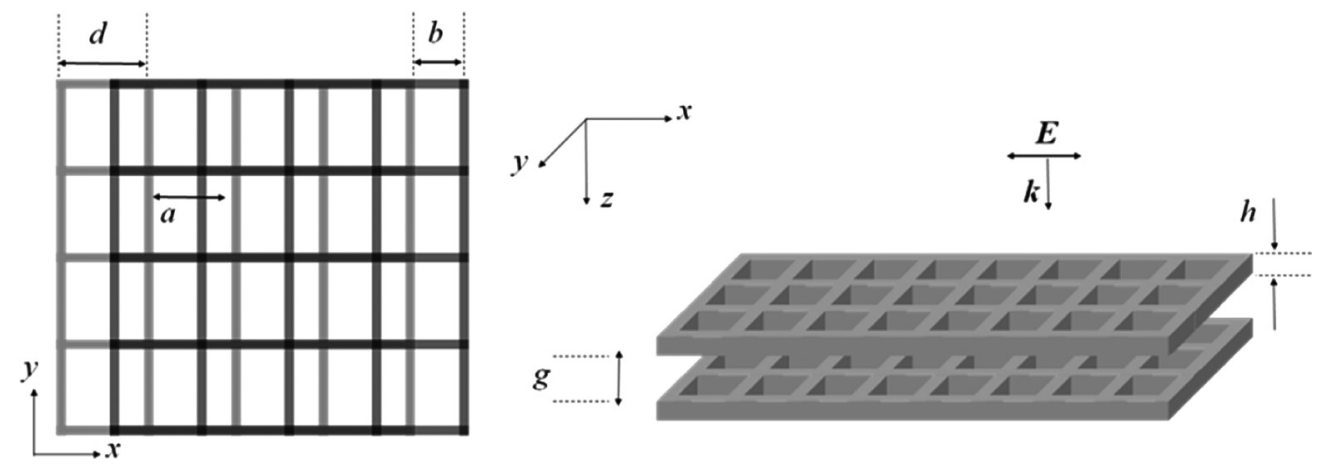

FIG. 1. Schematic of the investigated geometry; 2D square array of square holes (unshaded) in a PEC sheet (shaded).

meshes by $\varepsilon_{\mathrm{d}}$, and the field amplitude by $A^{m, n}$. For the vacuum region above the mesh, there will be an additional term for the incident field.

Within the mesh layer, the electric fields are only present in the square holes, and they may be expanded as a sum of waveguide modes [Eqs. (5)-(9)],

$$
\begin{aligned}
E_{x}^{\mathrm{cav}}= & \sum_{s, t}\left[B_{x}^{s, t} \psi_{2}(x, y) \exp \left(i q_{z}^{s, t} z\right)\right. \\
& \left.+C_{x}^{s, t} \psi_{2}(x, y) \exp \left(-i q_{z}^{s, t} z\right)\right], \\
E_{y}^{\mathrm{cav}}= & \sum_{s, t}\left[B_{y}^{s, t} \psi_{3}(x, y) \exp \left(i q_{z}^{s, t} z\right)\right. \\
& \left.+C_{y}^{s, t} \psi_{3}(x, y) \exp \left(-i q_{z}^{s, t} z\right)\right],
\end{aligned}
$$

where

$$
\begin{aligned}
& \psi_{2}(x, y)=\cos \left(\frac{s \pi x}{a}\right) \sin \left(\frac{t \pi y}{a}\right), \\
& \psi_{3}(x, y)=\sin \left(\frac{s \pi x}{a}\right) \cos \left(\frac{t \pi y}{a}\right),
\end{aligned}
$$

and

$$
q_{z}^{s, t}=\sqrt{\varepsilon_{\mathrm{h}}\left(\frac{\omega}{c}\right)^{2}-\left(\frac{s \pi}{a}\right)^{2}-\left(\frac{t \pi}{a}\right)^{2}} .
$$

The order of the waveguide modes is denoted by $s$ and $t$ and the associated propagation constant is given by $q_{z}^{s, t}$, the square hole side length is denoted by $a$, and the permittivity within the hole is denoted by $\varepsilon_{\mathrm{h}}$. The field amplitudes are denoted by $B_{i}^{s, t}$ and $C_{i}^{s, t}$. Manipulating and solving Maxwell's equations for the above field equations yield analytic solutions for the complex transmission and reflection amplitude coefficients,

$$
\begin{aligned}
& r^{m, n}=\sqrt{\left(A_{x}^{m, n}\right)^{2}+\left(A_{y}^{m, n}\right)^{2}}, \\
& t^{p, q}=\sqrt{\left(D_{x}^{p, q}\right)^{2}+\left(D_{y}^{p, q}\right)^{2}} .
\end{aligned}
$$

A full description of this method for a single mesh layer has been given previously ${ }^{14,17}$ and is not repeated here. The method is extended for the double mesh system by describing the electric fields in the additional regions in the same way with the fields in the holes of the second mesh layer also expanded as a sum of waveguide modes, while in the region between the meshes the fields are represented as a Fourier-Floquet expansion of the diffracted orders. To account for the multiple reflections between the meshes, two expansions are needed in this dielectric cavity [i.e., both an $\exp \left(-i k_{z}^{m, n} z\right)$ and an $\exp \left(i k_{z}^{m, n} z\right)$ sum]. The boundary conditions and continuity requirements then need to be applied at each boundary, resulting in pairs of coupled simultaneous equations. These are then solved, as before, to provide equations for the amplitude coefficients. The method is essentially the same as for the single layer, albeit more computationally laborious.

\section{RESULTS AND DISCUSSION}

Analysis of the mathematics for the transmission and reflection coefficients shows that the relationship between the resonance of each mesh, the resonance of the cavity between the meshes, and the relative alignment of the meshes is a complex one. However, despite the complexity of the equation, it is still possible to extract further insight into these relationships. For simplicity here, we have only considered incident radiation polarized parallel to the displacement direction, although the method can be used equally well to consider radiation perpendicular to it. From previous work, it is known that the transmission maximum for each mesh occurs individually for the $\{0, n\}$ diffracted orders when $\omega / c \approx$ $\sqrt{2\left[\left(k_{x}\right)^{2}+2(n \pi / d)^{2}\right]}$ (see Ref. 17). As we are at normal incidence, this reduces to $\omega / c \approx 2 n \pi / d$. Also, as before, only the first-order waveguide modes need to be included in the calculation as it is purely a "matching condition" across the interfaces, and therefore the first-order mode is sufficient to provide an accurate representation of the behavior. In addition, since the resonances occur in the nondiffracting regime, only the specular propagating beam (zero-order) in transmission below the second mesh needs to be considered. Applying these conditions gives the following general equation for the transmission amplitude coefficient:

$$
t^{0,0}=\frac{4 a^{2} G_{x} e^{i(g+2 h) q_{z}}}{d^{2} \pi}
$$

where $G_{x}$ is the complex amplitude coefficient incorporating the "overlap integrals" describing the boundary and orthogonality conditions and the alignment of the meshes. By further examination of this amplitude coefficient, we are able to isolate the terms involving the misalignment of the meshes, $b$, and the 
separation between them, $g$,

$$
G_{x}=\left(\frac{k_{z}^{0} \frac{4 a^{2}}{\pi}+C_{x}\left(q_{z} \frac{a^{2}}{2}-F_{1}+\exp \left[-2 i h q_{z}\right]\right)}{\exp \left[i(h+g) q_{z}\right]\left(q_{z} \frac{a^{2}}{2}-H_{1}+H_{3}-\frac{\exp \left[2 i h q_{z}\right]\left(q_{z} \frac{a^{2}}{2}-I_{1}\right)\left(q_{z} \frac{a^{2}}{2}+H_{1}-H_{3}\right)}{I_{1}+q_{z} \frac{a^{2}}{2}}\right)}\right) \frac{2 H_{4} \exp \left[i h q_{z}\right]}{q_{z} \frac{a^{2}}{2}+F_{1}},
$$

where

$$
\begin{gathered}
F_{1}=\sum_{m, n} Q_{a}^{m, n, 1,0} Q_{a}^{m, n, 1,0 *}, \\
H_{1}=\sum_{p, r} \frac{Q_{b}^{p, r, 1,0} Q_{b}^{p, r, 1,0 *} \exp \left[2 i g k_{z}^{p, r}\right]}{\exp \left[2 i g k_{z}^{p, r}\right]-1}, \\
H_{3}=-\sum_{p, r} \frac{Q_{b}^{p, r, 1,0} Q_{b}^{p, r, 1,0 *}}{\exp \left[2 i g k_{z}^{p, r}\right]-1}, \\
H_{4}=\sum_{p, r} \frac{Q_{a}^{p, r, 1,0} Q_{b}^{p, r, 1,0 *} \exp \left[-i g k_{z}^{p, r}\right]}{\exp \left[-2 i g k_{z}^{p, r}\right]-1}, \\
I_{1}=\sum_{u, v} Q_{b}^{u, v, 1,0} Q_{b}^{u, v, 1,0 *},
\end{gathered}
$$

and the overlap integral components, $Q_{i}$, are given by

$$
\begin{aligned}
Q_{a}^{m, n, 1,0}= & \int_{0}^{a} \int_{0}^{a} \exp \left[-i\left(k_{x}+\frac{2 m \pi}{d}\right) x\right] \\
& \times \exp \left[-i\left(k_{y}+\frac{2 n \pi}{d}\right) y\right] \sin \left[\frac{\pi y}{a}\right] d x d y, \\
Q_{b}^{m, n, 1,0}= & \int_{0}^{a} \int_{b}^{a+b} \exp \left[-i\left(k_{x}+\frac{2 m \pi}{d}\right) x\right] \\
& \times \exp \left[-i\left(k_{y}+\frac{2 n \pi}{d}\right) y\right] \sin \left[\frac{\pi y}{a}\right] d x d y .
\end{aligned}
$$

Examination of Eq. (13) shows that it is the $H_{4}$ term that contains the overlap integral components that describe the interaction between the two meshes via the evanescent fields, i.e., this term is affected by the relative misalignment of the meshes. The separation of the meshes, $g$, is clearly a dominant parameter within $G_{x}$. In particular, as $g$ increases, terms $H_{1}$ and $\mathrm{H}_{3}$ will increase rapidly. In other words, as the denominator (separation term) increases, the numerator (alignment term) becomes less dominant.

Figure 2 compares normal incidence transmission plots for both aligned and misaligned arrangements of two identical meshes for three different mesh separations. The meshes have a pitch of $d=10 \mathrm{~mm}$ and the sides of the holes are $a=4.5 \mathrm{~mm}$. For the misaligned geometry, a misalignment of $b=5 \mathrm{~mm}$ is illustrated. It is clear that when the separation of the meshes, $g$, is smaller than the wavelength, there is a significant difference between the transmission of the aligned and misaligned systems. In the aligned case when $g=0.1$ $\mathrm{mm}$ [Fig. 2(a)], the Fano-shaped resonance around $26.5 \mathrm{GHz}$ is in the wings of a coupled surface wave mode at $\sim 28 \mathrm{GHz}$. The former is a symmetric cosh-like mode, while the latter is
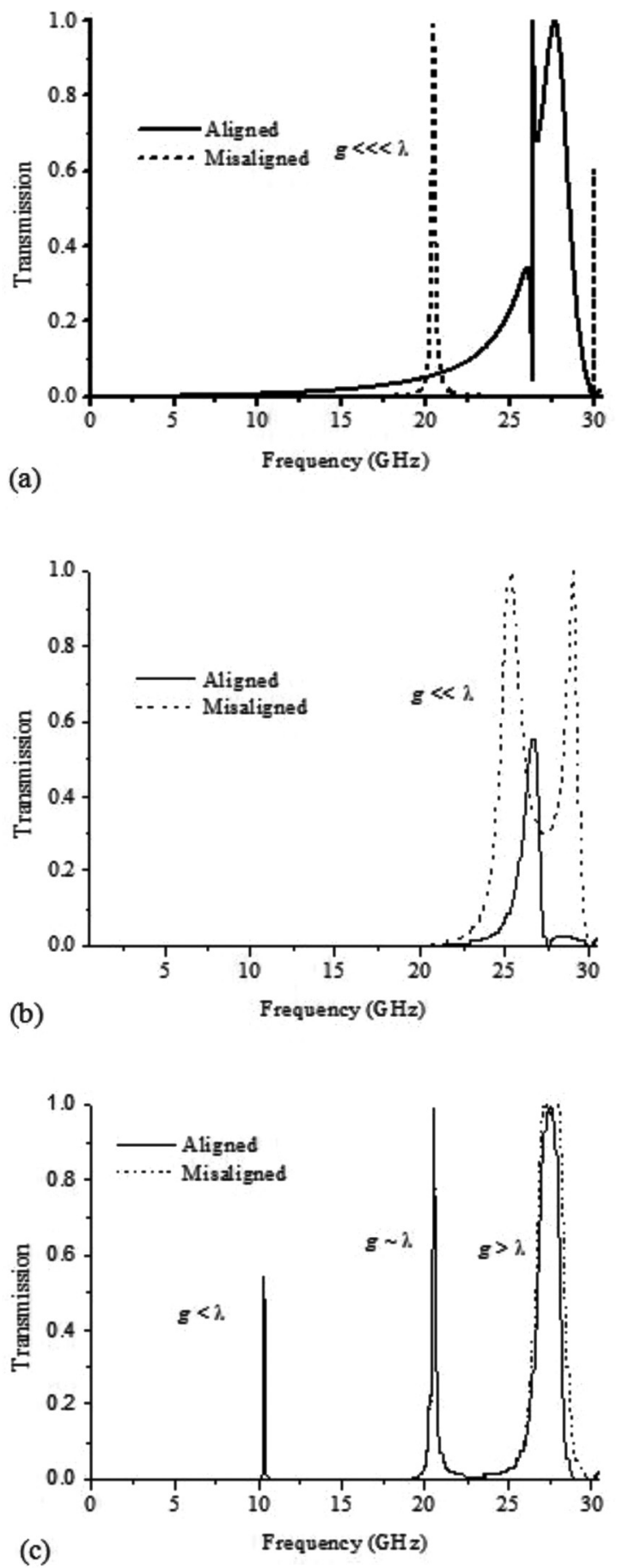

FIG. 2. Transmission for $d=10 \mathrm{~mm}, a=4.5 \mathrm{~mm}$ for (a) $g=0.1 \mathrm{~mm}$, (b) $g=4 \mathrm{~mm}$, and (c) $g=14 \mathrm{~mm}$. Each graph shows the results for both the aligned system and when the meshes are misaligned by $5 \mathrm{~mm}$ with respect to each other. 

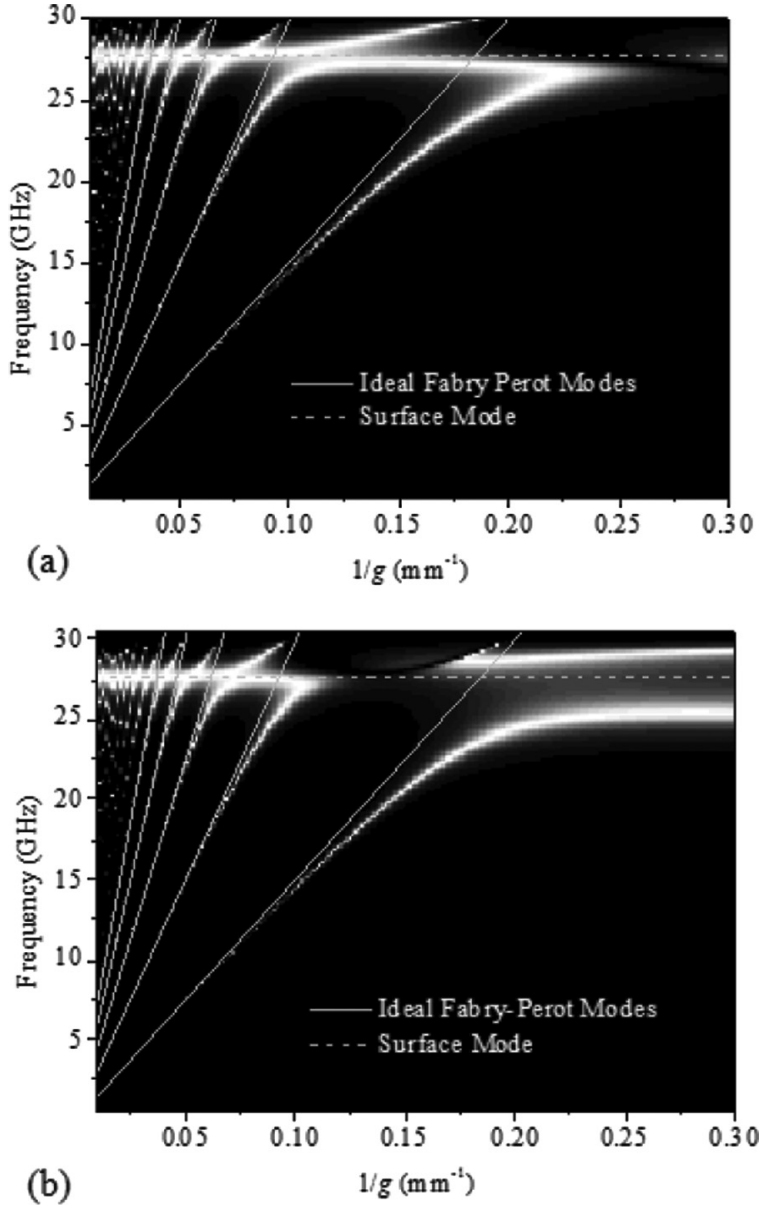

FIG. 3. Gray-scale plot of normal incidence transmission as a function of frequency and mesh separation with pitch $10 \mathrm{~mm}$ and hole sides $4.5 \mathrm{~mm}$ for (a) $b=0 \mathrm{~mm}$ and (b) $b=5 \mathrm{~mm}$. The ideal FabryPérot modes refer to the half-wavelength condition (i.e., $\lambda=2 d / n$ for $n=1,2,3 \ldots)$.

an antisymmetric sinh-like mode. In contrast, for the $5 \mathrm{~mm}$ misaligned case, the cosh-like (symmetric) mode resulting from the coupling between the surface mode of each mesh is present at $20 \mathrm{GHz}$, and the sinh-like mode has pushed up in frequency to just below the onset of diffraction at $30 \mathrm{GHz}$. Both of the modes supported by the double mesh structures arise from an interaction between a (symmetric/antisymmetric) pair of coupled surface waves and either the cavity's standing-wave resonance or the single mesh resonance to form a hybrid mode. This cosh-like and sinh-like coupling has distinct parallels to thin-film optical waveguides (otherwise known as metal-insulator-metal waveguides, or MIMs). MIMs support long-range surface plasmons that are bound to the surface and exhibit mode splitting due to symmetric and antisymmetric electric field distributions. ${ }^{24-26}$

In the long-wavelength limit, each hybrid mode has strong Fabry-Pérot characteristics and approaches the frequency where, in an ideal system, the optical path length between each partial mirror (mesh) is equal to an integer number of half-wavelengths. Conversely, in the short-wavelength limit, each hybrid mode is dominated by the single mesh resonance and displays strong surface wave characteristics, as will be shown later.

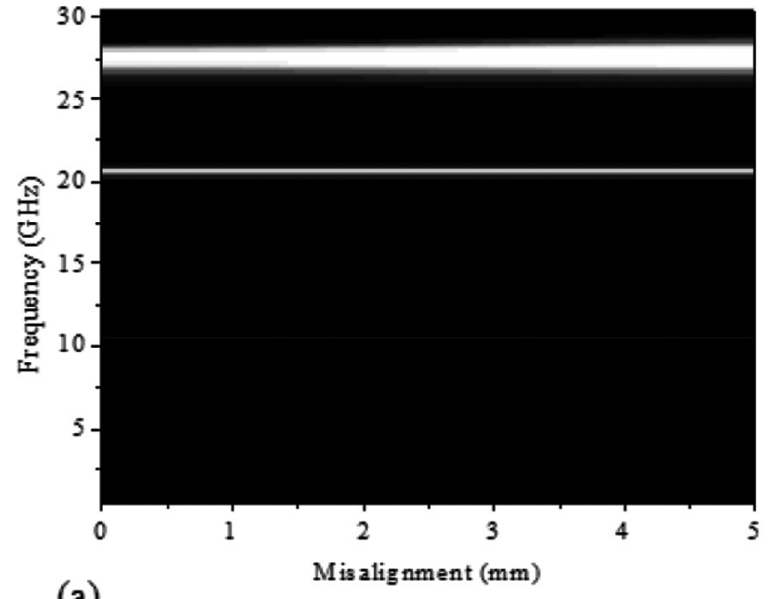

(a)

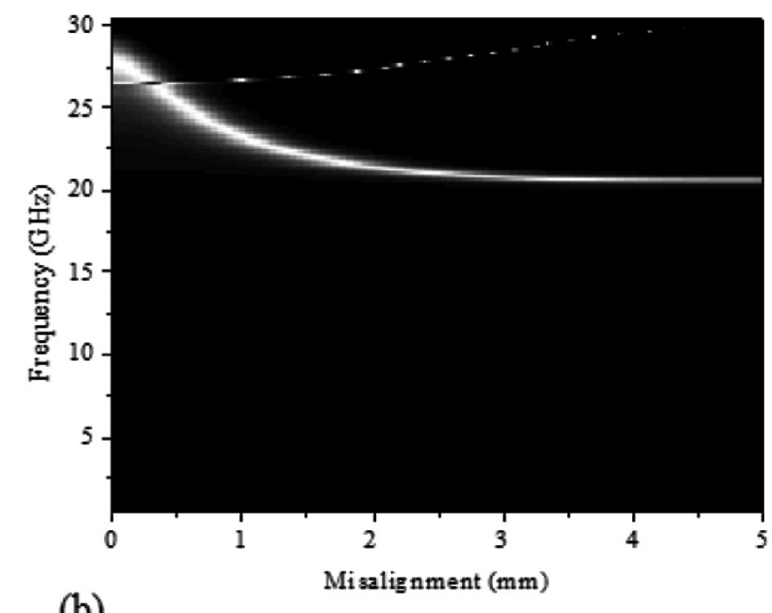

(b)

FIG. 4. Gray-scale plot of normal incidence transmission as a function of frequency and misalignment with pitch $10 \mathrm{~mm}$ and hole sides $4.5 \mathrm{~mm}$ for (a) mesh separation $14 \mathrm{~mm}$ and (b) mesh separation $0.1 \mathrm{~mm}$.

As the separation between the meshes increases to approach the wavelength, the responses for both the aligned and misaligned systems become increasingly similar, as shown by Figs. 2(b) and 2(c). Figure 2(c) shows reasonable qualitative agreement for the region where $g>\lambda$, and we might anticipate that the similarity between the aligned and misaligned responses would worsen for lower frequencies as the wavelength increases to become comparable to and then greater than $g$. However, this regime, where the wavelength is greater than $g$, is far from the onset of diffraction, and evanescent diffraction has little significance. Thus, with no real propagating diffracted orders, the microscopic details describing the subwavelength structure are not conveyed from one layer to the other, including the misalignment of the meshes with respect to each other, and therefore this misalignment has little effect on the response of the system. In other words, each mesh can be effectively described as a homogeneous medium.

Figure 3 shows a more extensive, gray-scale plot of the normal incidence transmission as a function of frequency and mesh separation, again for both aligned and misaligned systems. A family of modes between the two meshes can be clearly observed. At low frequencies, each mode is 

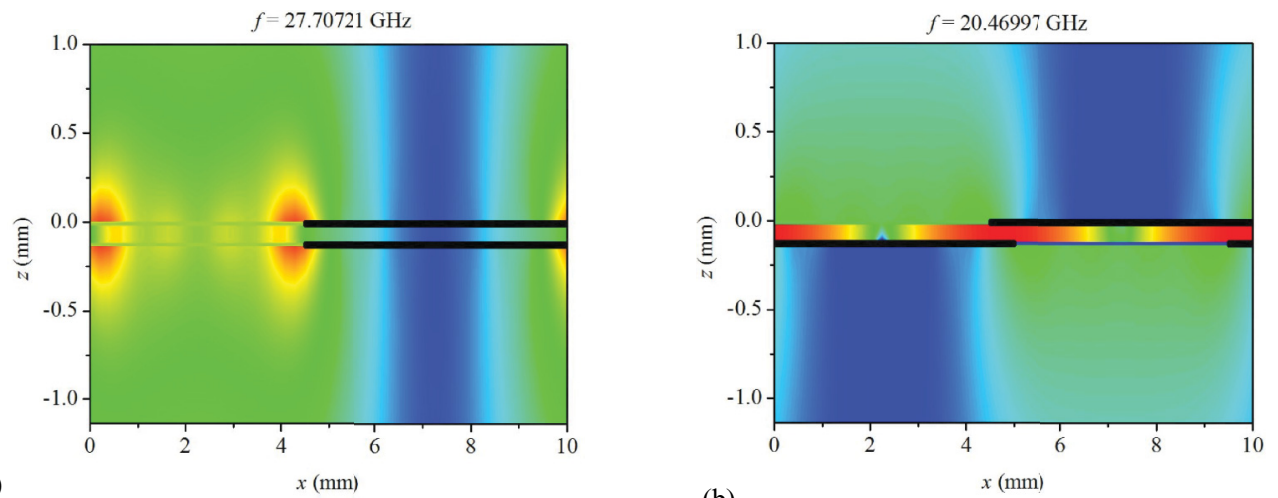

(a)

(b)
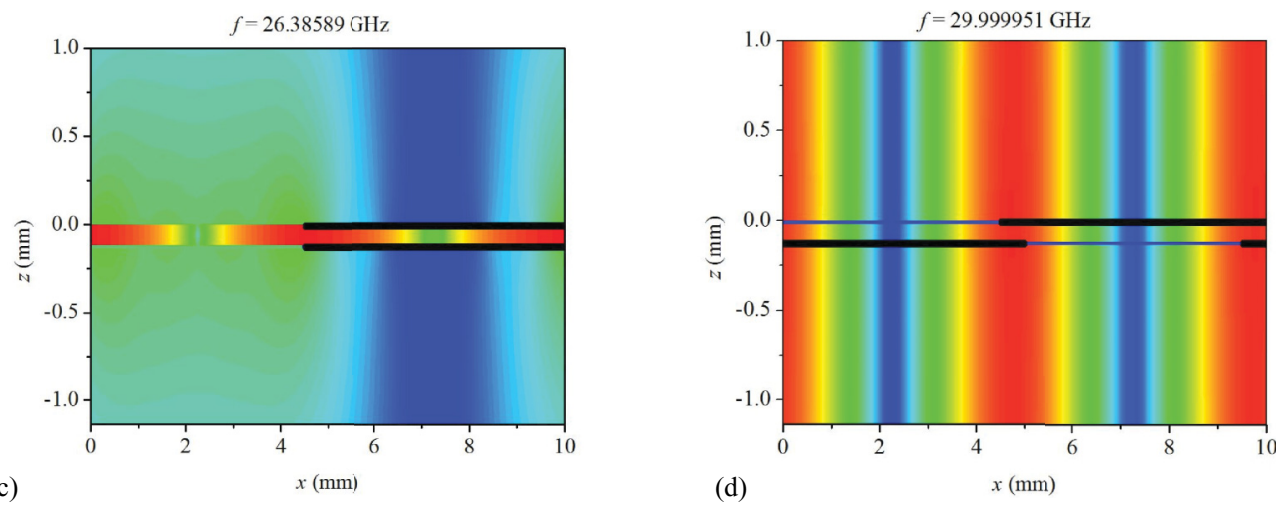

High negative field

High positive field

FIG. 5. (Color online) Complex magnitude $\mathbf{E}$ field plots in the $X Z$ plane for a double-mesh-layer system of pitch $d=10 \mathrm{~mm}$, hole side length $a=4.5 \mathrm{~mm}$, and mesh separation $g=0.1 \mathrm{~mm}$ at (a) $27.7 \mathrm{GHz}$ with misalignment $b=0 \mathrm{~mm}$; (b) $20.4 \mathrm{GHz}$ with misalignment $b=5 \mathrm{~mm}$; (c) $25.5 \mathrm{GHz}$ with misalignment $b=0 \mathrm{~mm}$; and (d) $29.9 \mathrm{GHz}$ with misalignment $b=5 \mathrm{~mm}$. The metal meshes are represented by thick black lines.

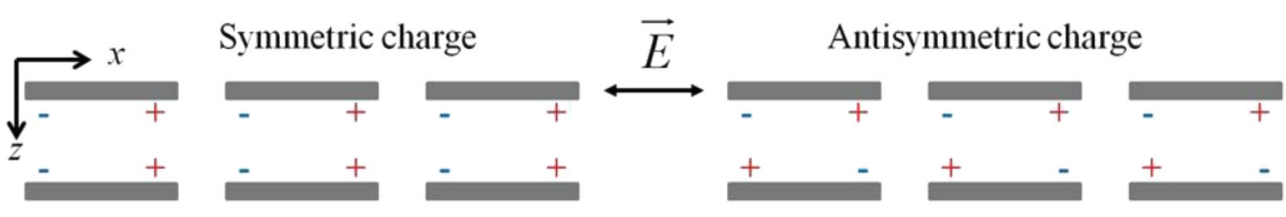

(a)

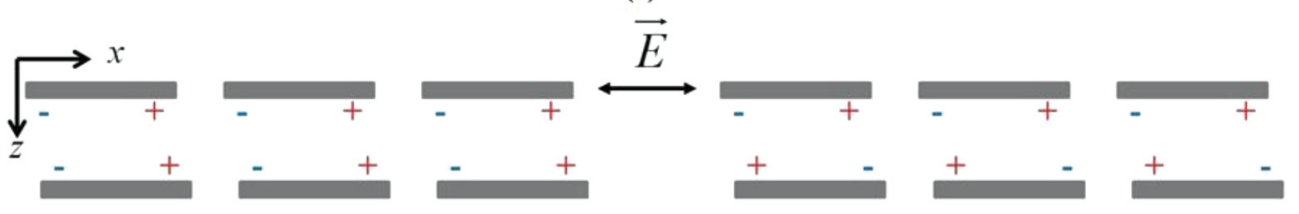

(b)

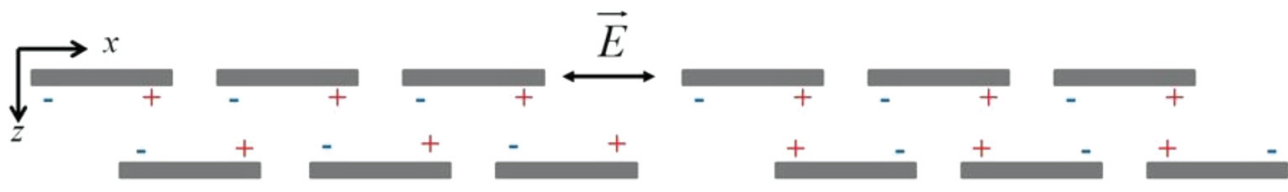

(c)

FIG. 6. (Color online) Schematic of possible charge distributions for a double layer of identical metal slit arrays (one-dimensional grids) when the layers are (a) aligned with respect to each other; (b) slightly misaligned with respect to each other, and (c) at maximum misalignment with respect to each other. 
Fabry-Pérot-like; however, as the frequency approaches that of the surface mode supported by a single mesh layer of identical geometry, hybridization occurs, as is strongly evidenced by the perturbation of the modes from the simple half-integer wavelength condition of the ideal Fabry-Pérot resonance for perfect electrical mirrors. The transmission plots in Fig. 2 are line plots of Fig. 3 at specific separations; however, Fig. 2(a) is beyond the range of Fig. 3. The sinh-like and cosh-like modes discussed previously can be observed separating as $1 g$ increases (i.e., as the separation, $g$, decreases).

In Fig. 4, the transmission is plotted as a function of frequency and misalignment for separations of 14 and 0.1 mm [Figs. 4(a) and 4(b), respectively]. Figure 4(b) shows the behavior of the two modes as the misalignment between two meshes with squares of side length $4.5 \mathrm{~mm}$ is increased. When the two meshes are exactly aligned $(b=0 \mathrm{~mm})$, a broad mode is seen at approximately $26.5 \mathrm{GHz}$, with a much sharper mode slightly lower in frequency at $25.5 \mathrm{GHz}$. As the misalignment increases, the modes cross, with the sharper mode moving up toward the onset of diffraction at $30 \mathrm{GHz}$ and the broad mode dropping down to approximately $20 \mathrm{GHz}$ at maximum misalignment $(b=5 \mathrm{~mm})$. Exploration of the magnitude of the electric fields $\left(\boldsymbol{E}_{z}\right)$, calculated using the modal-matching method, both between and outside the meshes as the misalignment varies, reveals that while the fields outside of the meshes remain reasonably constant, the fields between the meshes show greater enhancement as the misalignment is increased (not shown). The electric field between the two mesh layers is given by

$$
\begin{aligned}
E_{z}^{\text {between }}= & \sum_{p, r}\left[F_{x}^{p, r}\left(G_{x}\right) \psi_{1}^{p, r}(x, y) \exp \left(-i k_{z}^{m, n} z\right)\right. \\
& \left.-D_{x}^{p, r}\left(G_{x}\right) \psi_{1}^{p, r}(x, y) \exp \left(i k_{z}^{m, n} z\right)\right],
\end{aligned}
$$

where $F_{x}^{p, r}$ and $D_{x}^{p, r}$ describe complex amplitude coefficients and are functions of $G_{x}$.

The field enhancement is expected to be greatest at the edges of the holes due to the evanescent diffraction. At zero misalignment, the holes in both meshes are directly in line with each other and the field enhancement between the meshes is localized in a small area at the edge of the holes [Figs. 5(a) and 5(c)]. As the misalignment between the meshes gradually increases, the alignment terms in $G_{x}\left(H_{4}\right)$ start to become dominant, increasing the field enhancement between the meshes, which in turn increases the coupling to the resonance on the opposite mesh [Figs. 5(b) and 5(d)].
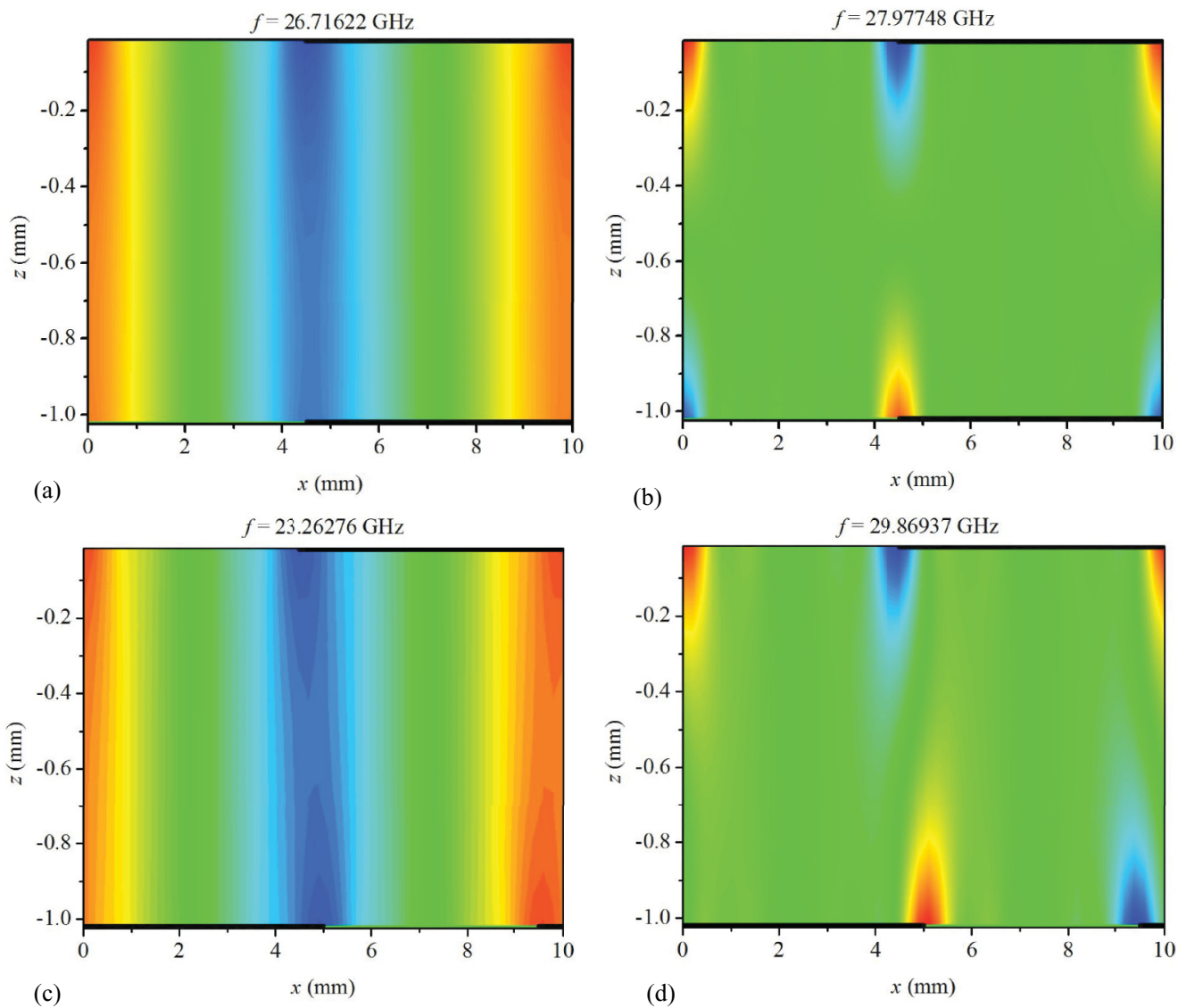

(b)
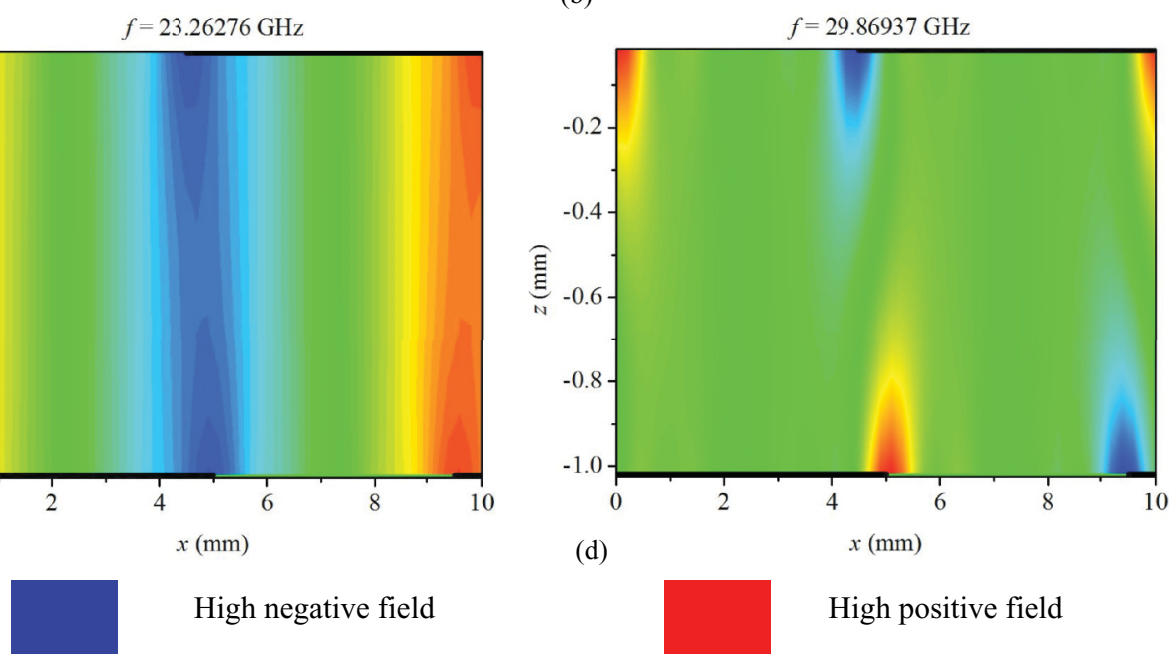

FIG. 7. (Color online) Directional $\mathbf{E}$ field plots in the $X Z$ plane in the region between the two meshes for a double-mesh-layer system of pitch $d=10 \mathrm{~mm}$, hole side length $a=4.5 \mathrm{~mm}$, and mesh separation $g=1 \mathrm{~mm}$ at (a) $26.7 \mathrm{GHz}$ with misalignment $b=0 \mathrm{~mm}$; (b) $28.0 \mathrm{GHz}$ with misalignment $b=0 \mathrm{~mm}$; (c) $23.3 \mathrm{GHz}$ with misalignment $b=5 \mathrm{~mm}$; and (d) $29.9 \mathrm{GHz}$ with misalignment $b=5 \mathrm{~mm}$. The metal meshes are represented by thick black lines. 
By considering the charge distribution for the simpler, well understood case of a similarly spaced pair of identical metal slot arrays (i.e., one-dimensional "grids"), further understanding of these modes can be gained. In this simpler case, there are two possible charge configurations, i.e., symmetric and antisymmetric [Fig. 6(a)]. The symmetric charge configuration produces an antisymmetric (sinh-like) electric field configuration and can be considered as surface-mode-like due to the high electric fields being bound along the interfaces. In contrast, the antisymmetric charge configuration results in a symmetric (cosh-like) electric field configuration and the high electric fields occur within the cavity between the two meshes and are not bound to the surfaces and therefore can be considered as Fabry-Pérot-mode-like. When the slot arrays are laterally misaligned with respect to each other, these charge configurations begin to be perturbed. The surface-mode-like configuration develops Fabry-Pérot characteristics and vice versa [Fig. 6(b)]. The greater the misalignment, the more the charge configuration is perturbed. At maximum misalignment, there are still two possible configurations but they both have the same energy and they have both Fabry-Pérot and surface mode characteristics [Fig. 6(c)]. However, for the double mesh system investigated here, when the holes are laterally misaligned, the fourfold symmetry of the aligned unit cell is reduced to a single plane of symmetry. In addition, there are regions of the unit cell that are unaffected by the lateral misalignment, and therefore, for this structure, the two configurations are not equal in energy. Figure 7 shows the directional electric fields in region III (between the two meshes) for the structure with $1 \mathrm{~mm}$ separation between the meshes. When the meshes are aligned, the symmetric charge configuration (Fabry-Pérot-like mode) is evident for the sharp mode [Fig. 7(a)], while the antisymmetric configuration of the surfacelike mode is shown for the broad mode [Fig. 7(b)]. When the meshes are laterally misaligned with respect to each other by $5 \mathrm{~mm}$, the hybrid symmetric (with respect to the charge configuration) mode [Fig. 7(c)] and the antisymmetric hybrid mode [Fig. 7(d)] corresponding to the charge configurations in Fig. 6(c) are illustrated.

\section{CONCLUSION}

In conclusion, using an adapted modal-matching method, a complete analytical solution for transmission through a double layer of PEC mesh with dielectric spacing has been developed by extending a previous method. ${ }^{17}$ Examination of the numerical results and analytic equations shows that the EM response of the system is highly dependent on the strength of the evanescent diffraction. Families of Fabry-Pérot-like modes are supported, and as these approach the frequency of the surface mode supported by a single mesh layer, the two modes interact forming a hybrid mode. The solution shows that it is the separation between the mesh layers that is the critical parameter, while the misalignment of the meshes with relation to each other is only significant in the regime where the separation is less than the wavelength. Exploration of the analytical expressions obtained for the transmission through the structure and the field profiles gives insight into the behavior of the observed resonances by determining the conditions in which the alignment terms become dominant. In particular, in the case of closely spaced layers, as the lateral misalignment of the meshes with respect to each other increases, the perturbation of the charge distributions on each mesh causes greater enhancement of the electric field between the two meshes.

\section{ACKNOWLEDGMENTS}

M.C.T. would like to thank BAE Systems, Ltd. for their financial support, and in particular Dr. Sajad Haq for helpful discussions.
${ }^{1}$ F. J. Garcia de Abajo, Rev. Mod. Phys. 79, 1267 (2007).

${ }^{2}$ R. Ulrich, Infr. Phys. 7, 65 (1967).

${ }^{3}$ T. W. Ebbesen, H. J. Lezec, H. F. Ghaemi, T. Thio, and P. A. Wolff,

Nature (London) 391, 667 (1998).

${ }^{4}$ U. Fano, J. Opt. 31, 213 (1941).

${ }^{5}$ R. Ulrich, Infr. Phys. 7, 37 (1967).

${ }^{6}$ J. B. Pendry, L. Martin-Moreno, and F. J. Garcia-Vidal, Science 305, 847 (2004).

${ }^{7}$ R. Ulrich and M. Tacke, Appl. Phys. Lett. 22, 251 (1972).

${ }^{8}$ L. B. Whitbourn and R. C. Compton, Appl. Opt. 24, 217 (1985).

${ }^{9}$ R. C. Compton, IEEE Trans. Microwave Theory Tech. 33, 1083 (1985).

${ }^{10}$ J. H. Coggon, Geophysics 36, 132 (1971).

${ }^{11}$ L. F. Shen, X. D. Chen, and T. J. Yang, Opt. Express 16, 3326 (2008).

${ }^{12}$ F. J. Garcia-Vidal, L. Martin-Moreno, and J. B. Pendry, J. Opt. A 7, S97 (2005).

${ }^{13}$ J. Bravo-Abad, L. Martin-Moreno, F. J. Garcia-Vidal, E. Hendry, and J. G. Rivas, Phys. Rev. B 76, 241102 (2007).

${ }^{14}$ E. Hendry, A. P. Hibbins, and J. R. Sambles, Phys. Rev. B 78, 235426 (2008).
${ }^{15}$ J. Bravo-Abad, F. J. Garcia-Vidal, and L. Martin-Moreno, Phys. Rev. Lett. 93, 227401 (2004).

${ }^{16}$ R. C. Mcphedran, G. H. Derrick, and L. C. Botten, Electromagnetic Theory of Gratings (Springer-Verlag, Berlin, 1980).

${ }^{17}$ M. C. Taylor, J. D. Edmunds, E. Hendry, A. P. Hibbins, and J. R. Sambles, Phys. Rev. B 82, 155105 (2010).

${ }^{18}$ Z. N. Yu, P. Deshpande, W. Wu, J. Wang, and S. Y. Chou, Appl. Phys. Lett. 77, 927 (2000).

${ }^{19}$ R. Ortuno, C. Garcia-Meca, F. J. Rodriguez-Fortuno, J. Marti, and A. Martinez, Phys. Rev. B 79, 075425 (2009).

${ }^{20}$ C. Cheng, J. Chen, Q. Y. Wu, F. F. Ren, J. Xu, Y. X. Fan, and H. T. Wang, Appl. Phys. Lett. 91, 11111 (2007).

${ }^{21}$ M. D. He, L. L. Wang, J. Q. Liu, X. Zhai, Q. Wan, X. Chen, and B. S. Zou, Appl. Phys. Lett. 93, 103109 (2008).

${ }^{22}$ F. Miyamaru and M. Hangyo, Phys. Rev. B 71, 165408 (2005).

${ }^{23}$ Z. Marcet et al., Opt. Lett. 35, 2124 (2010).

${ }^{24}$ J. Chen, G. A. Smolyakov, S. R. J. Brueck, and K. J. Malloy, Opt. Express 16, 14902 (2008).

${ }^{25}$ R. Zia, M. D. Selker, P. B. Catrysse, and M. L. Brongersma, J. Opt. Soc. Am. A 21, 2442 (2004).

${ }^{26}$ J. A. Dionne, L. A. Sweatlock, H. A. Atwater, and A. Polman, Phys. Rev. B 73, 035407 (2006). 